\begin{tabular}{cc|c}
\hline Tar. Bil. Der. & Journal of Agricultural Sciences \\
& $\begin{array}{c}\text { Dergi web sayfası: } \\
\text { www.agri.ankara.edu.tr/dergi }\end{array}$ & Journal homepage: \\
& www.agri.ankara.edu.tr/journal
\end{tabular}

\title{
Physicochemical, Functional and Microbiological Properties of Hardaliye Beverages Produced from Different Grapes and Collected from Different Households
}

\author{
Fatma COSKUN ${ }^{\mathrm{a}}$, Muhammet ARICI ${ }^{\mathrm{b}}$, Mehmet GULCU ${ }^{\mathrm{c}}$, Gulnaz CELIKYURT ${ }^{\mathrm{d}}$, Mustafa MIRIK

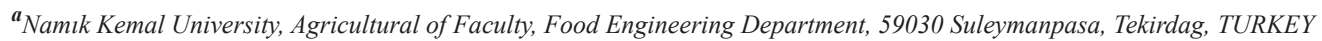

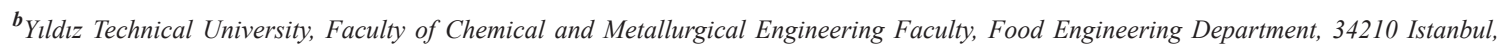 \\ TURKEY \\ ${ }^{c}$ Tekirdag Viticultural Research Station, 59100 Suleymanpasa, Tekirdag, TURKEY

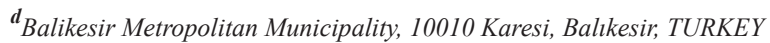

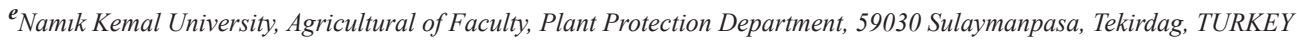

\section{ARTICLE INFO}

Research Article DOI: $10.15832 /$ ankutbd.446458

Corresponding Author: Fatma COSKUN, E-mail: fcoskun@nku.edu.tr, Tel: +90 (282) 2502162

Received: 17 Novemver 2014, Received in Revised Form: 31 May 2017, Accepted: 07 June 2017

\section{ABSTRACT}

Twenty-three hardaliye beverage samples were collected from different regions of Kirklareli city in Turkey and five hardaliye samples were produced under laboratory conditions according to traditional methods. Total phenolic compounds, monomeric anthocyanins (malvidin-3-glucoside), volatile acid, ethyl alcohol, total sugar contents, total acidity (lactic acid), $\mathrm{pH}$, color values ( $L$ (brightness), $a$ (red-green), $b$ (yellow-blue)), total mesophilic aerobic bacteria, lactic acid bacteria, yeast and mold and coliform counts were determined. Acidity between 0.38 and $0.91 \%, \mathrm{pH}$ from 3.54 and 4.33, color (L.a.b.) values from 7.57 to 13.74 , from 0.71 to 7.68 , from 0.73 to 4.50 , respectively were determined. Total phenolics from 368.8 to $2647.5 \mathrm{mg} \mathrm{L}^{-1}$, ethanol between 0.4 and $6.0 \%$ in 7 out of 23 examples, TMAB number $3 \times 10^{1}$ $3.2 \times 10^{6} \mathrm{cfu} \mathrm{mL} \mathrm{m}^{-1}$, LAB $1 \times 10^{2}-3 \times 10^{5} \mathrm{cfu} \mathrm{mL}^{-1}$ in 16 out of 23 samples, yeast and mold count $1 \times 10^{1}-2,3 \times 10^{4} \mathrm{cfu} \mathrm{mL}^{-1}$ in 18 out of 23 samples were found. According to the results obtained, coliforms and Escherichia coli were not found in the analyzed samples. Statistical analysis results revealed that differences between samples were significant $(\mathrm{P} \leq 0.01)$. Given its bioactive characteristics, hardaliye beverage should be produced at large-scale and be recognized by large communities. Keywords: Fermentation; Grape juice; Hardaliye; Mustard seeds; Traditional beverage

\section{Farklı Evlerden Toplanan ve Farklı Üzümlerden Üretilen Hardaliye Örneklerinin Fizikokimyasal, Fonksiyonel ve Mikrobiyolojik Özellikleri}

\section{ESER BİLGISI}

Araştırma Makalesi

Sorumlu Yazar: Fatma COSKUN, E-posta: fcoskun@nku.edu.tr, Tel: +90 (282) 2502162

Geliş Tarihi: 17 Kasım 2014, Düzeltmelerin Gelişi: 31 Mayıs 2017, Kabul: 07 Haziran 2017 


\section{ÖZET}

$\mathrm{Bu}$ araştırmada, Kırklareli’nin farklı bölgelerinden yirmi üç hardaliye örneği toplanmış ve laboratuvar şartlarında geleneksel metotlara göre beş adet hardaliye üretilmiştir. Bu örneklerde toplam fenolik madde, monomerik antosiyanin (malvidin-3-glikozit), uçucu asit, etil alkol, invert şeker, toplam asitlik (laktik asit cinsinden), $\mathrm{pH}$, renk değerleri ( $L$ (parlaklık), $a$ (kırmızı-yeşil), $b$ (sarı-mavi)), toplam aerobik mezofil bakteri, laktik asit bakterileri, maya-küf ve koliform bakteri sayıları belirlenmiştir. Asitlik \% 0.38-0.91 arasında, pH 3.54-4.33 arasında, renk (L.a.b.) değerleri sırasıyla 7.57-13.74; 0.71-7.68; 0.73-4.50 olarak tespit edilmiştir. Toplam fenolik madde 368.8-2647.5 mg L $\mathrm{L}^{-1}$ arasında,

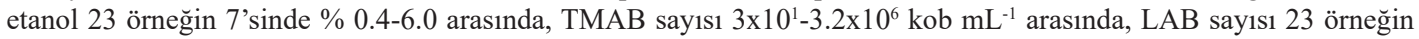
16 'sında ( 16 out of 23 samples) $1 \times 10^{2}-3 \times 10^{5} \mathrm{kob} \mathrm{mL}^{-1}$ arasında, maya-küf sayıs 23 örneğin $18^{\prime}$ inde (18 out of 23 samples) $1 \times 10^{1}-2.3 \times 10^{4} \mathrm{kob} \mathrm{mL} \mathrm{mL}^{-1}$ arasında bulunmuştur. Elde edilen sonuçlara göre, analiz edilen hiç bir örnekte koliform bakteri ve Escherichia coli tespit edilmemiştir. Analizler sonuçlarına göre örnekler arasındaki farklılıklar istatistiksel olarak önemli bulunmuştur $(\mathrm{P} \leq 0.01)$. Biyoaktif özellikleri dikkate alındığında, hardaliyenin büyük ölçekli üretiminin yapılması ve toplumun tanıması sağlanmalıdır.

Anahtar Kelimeler: Fermantasyon; Üzüm suyu; Hardaliye; Hardal tohumu; Geleneksel içecek

(C) Ankara Üniversitesi Ziraat Fakültesi

\section{Introduction}

Hardaliye is a lactic acid fermented beverage that is produced from red grape or grape juice with the addition of crushed mustard seeds and benzoic acid and it is widely consumed in the Thrace region of Turkey (Arici \& Coskun 2001). As well known, grape is rich fruit interms of phenolic compounds (Shahidi \& Naczk 1995). In vitro experimental systems also showed that flavonoids possess antiinflammatory, antiallergic, antiviral, and anticarcinogenic properties (Middleton 1998). Kanemaru \& Miyamoto (1990) detected that 0.8\% mustard extract showed bacteriostatic action against Staphylococcus aureus and Escherichia coli in culture medium. When essential oil of mustard was used, total mesophilic aerobic bacteria and lactic acid bacteria were significantly lower than the control after 2 days of storage (Lemay et al 2002). In a research conducted by Nielsen \& Rios (2000), allyl isothiocyanate, a bioactive substance of mustard, was shown to have an inhibitory effect against Penicillium commune, P. roqueforti, Aspergillus flavus and Endomyces fibuliger. They also determined minimum inhibitory concentration to range between 1.8 and $3.5 \mathrm{mg} \mathrm{mL}^{-1}$. Therefore, it is possible to say that the beneficial effects of hardaliye are not only related with grape which includes bioactive components but also with mustard which gained antibacterial and antifungal effects to hardaliye beverage.

The present study is composed of two parts. In the first part, 23 hardaliye beverage samples collected from local producers were analyzed in terms of their physicochemical, functional and microbiological properties. In the second part, fresh hardaliye beverage samples were produced from different grape types and their physicochemical and microbiological properties were investigated.

\section{Material and Methods}

\subsection{Material}

In this research, twenty-three hardaliye samples were collected from different households of Kirklareli city in Turkey. In the second stage, hardaliye samples were produced in the laboratory using different types of grapes. These grape types were Dingil Kara, Kara Uzum, Carbernet Sauvignon, Yerli Izabella and Siyah Uzum and procured from Tekirdag Viticulture Research Institute.

\subsection{Methods}

Grapes are washed and pressed in oak barrels which have a tap at the position of $10 \mathrm{~cm}$ above the bottom of the barrel. Then $0.2 \%$ of crushed raw mustard 
seed and $0.1 \%$ of sodium benzoate were added and the solution was left to fermentation at room temperature $\left(22^{\circ} \mathrm{C}\right)$ for 7 days.

The $\mathrm{pH}$ of the samples was measured using WTW 330 model $\mathrm{pH}$ meter with composite electrodes. Total acidity was determined according to the titration method reported by Cemeroğlu (2007). Total sugar contents of the samples were determined using Lane Eynon method (Cemeroğlu 2007). In order to determine total phenolic content of the samples, Folin-Ciocalteu spectrophotometric method was used (Singleton \& Rossi 1965). Monomeric anthocyanins were determined using the method reported by Cemeroğlu (2007). Alcohol contents of the samples were determined using ebulliometer (Jacobson 2006). Total volatile acid contents were determined using the method reported by Fidan (1975). The color of the samples was measured using a Hunter Lab colorimeter (Model D25 LT).

Standard methods defined by FDA/Bacteriological Analytical Manual for microbiological analysis were used for preparation of the samples for analysis. 25 $\mathrm{mL}$ hardaliye sample was added into Maximum Recovery Diluent(MRD) and it was homogenization. Other serial dilutions $\left(10^{-2}, 10^{-3}, 10^{-4}\right.$ and $\left.10^{-5}\right)$ were prepared using the initial dilution. These dilutions prepared were used for all microbiological analysis. Total bacteria were enumerated using plate count agar (PCA). For counting coliform bacteria, violet red bile agar (VRBA) and for counting E. coli, eosin methylene blue (EMB) agar were used. For counting yeast-mold, potato dextrose agar (PDA), was used. Numbers of total mesophilic aerobic bacteria, coliform bacteria, E. coli and yeast and mold were determined by providing the appropriate incubation temperature and duration (Anonymous 2001a; 2001b; 2001c; 2002). De Man Rogosa Sharpe (MRS) agar was used for determination of lactic acid bacteria (Baumgart 1993).

In order to determine differences between samples, one-way ANOVA was performed using SPSS 18.0 statistical package. All statistical analyses were performed according to completely randomized design (SPSS 2009).

\section{Results and Discussion}

Some physicochemical properties of hardaliye samples collected from different households are shown in Table 1. Some chemical properties of hardaliye samples collected from different households are shown in Table 2. As can be seen from the tables, remarkable differences between the values of total phenolic, monomeric anthocyanins and total sugar contents were observed. Differences between the samples were found to be significant $(\mathrm{P}<0.01)$. Some microbiological properties of hardaliye samples collected from different households are shown in Table 3.

In the second part of the study, the fresh hardaliye samples were produced from different grape types according to the traditional production method. Their physicochemical and chemical properties are presented in Tables 4 and 5. Differences between the samples were found to be significant $(\mathrm{P}<0.01)$. Total bacteria, lactic acid bacteria and yeast-mold counts of the samples are shown in Table 6. Coliforms and E. coli were not observed.

In this study, $\mathrm{pH}$ values of hardaliye samples collected from producers are between 3.54 and 4.33. Arici \& Coskun (2001) collected twentysix hardaliye samples from different spots of the Kirklareli city and the $\mathrm{pH}$ values determined in this study was similar to those determined in their study. $\mathrm{pH}$ values of samples produced in the laboratory are between 3.33 and 3.73 . These values were lower than $\mathrm{pH}$ value (4.42) reported by Coşkun et al (2009). Total acidity values obtained in these two studies are similar. Volatile acid values of all samples were similar or slightly higher than value $\left(0.25 \mathrm{~g} \mathrm{~L}^{-1}\right)$ reported by Coşkun et al (2009).

Total phenolics of anthocyanins in hardaliye samples collected from houses are generally higher than those produced in the laboratory. Diversity of grape varieties used in the production might have caused different anthocyanin and total phenolic contents in the samples. Unsuitable storage conditions, time and temperature causes 
Table 1- Some physicochemical properties of the hardaliye samples collected from different households

\begin{tabular}{cccccc}
\hline Sample & Acidity & \multirow{2}{*}{$\mathrm{N} H$} & \multicolumn{3}{c}{ Color } \\
\cline { 4 - 6 } no & $(\%)$ & & $L$ & $a$ & $b$ \\
\hline 1 & $0.84 \pm 0.02$ & $3.62 \pm 0.02$ & $7.78 \pm 0.02$ & $2.88 \pm 0.03$ & $2.59 \pm 0.01$ \\
2 & $0.49 \pm 0.01$ & $4.11 \pm 0.01$ & $7.79 \pm 0.04$ & $1.62 \pm 0.02$ & $2.10 \pm 0.00$ \\
3 & $0.63 \pm 0.02$ & $4.02 \pm 0.00$ & $7.86 \pm 0.01$ & $1.78 \pm 0.02$ & $0.47 \pm 0.00$ \\
4 & $0.59 \pm 0.02$ & $4.01 \pm 0.01$ & $8.46 \pm 0.01$ & $2.21 \pm 0.01$ & $1.27 \pm 0.02$ \\
5 & $0.49 \pm 0.06$ & $3.80 \pm 0.02$ & $8.67 \pm 0.02$ & $3.48 \pm 0.02$ & $1.79 \pm 0.01$ \\
6 & $0.42 \pm 0.01$ & $3.90 \pm 0.01$ & $8.57 \pm 0.02$ & $2.65 \pm 0.05$ & $1.44 \pm 0.04$ \\
7 & $0.56 \pm 0.04$ & $3.81 \pm 0.01$ & $7.57 \pm 0.02$ & $1.11 \pm 0.01$ & $0.85 \pm 0.05$ \\
8 & $0.70 \pm 0.03$ & $3.56 \pm 0.02$ & $9.80 \pm 0.02$ & $4.49 \pm 0.01$ & $1.86 \pm 0.01$ \\
9 & $0.56 \pm 0.01$ & $4.02 \pm 0.02$ & $12.09 \pm 0.01$ & $3.45 \pm 0.02$ & $4.33 \pm 0.01$ \\
10 & $0.42 \pm 0.02$ & $4.25 \pm 0.02$ & $10.77 \pm 0.02$ & $4.71 \pm 0.01$ & $2.36 \pm 0.01$ \\
11 & $0.35 \pm 0.00$ & $4.12 \pm 0.02$ & $11.45 \pm 0.02$ & $2.09 \pm 0.01$ & $1.96 \pm 0.01$ \\
12 & $0.38 \pm 0.02$ & $4.03 \pm 0.03$ & $11.81 \pm 0.01$ & $2.46 \pm 0.01$ & $3.77 \pm 0.02$ \\
13 & $0.42 \pm 0.01$ & $4.33 \pm 0.03$ & $12.49 \pm 0.02$ & $5.09 \pm 0.01$ & $4.50 \pm 0.05$ \\
14 & $0.49 \pm 0.02$ & $3.88 \pm 0.02$ & $13.74 \pm 0.02$ & $4.19 \pm 0.02$ & $4.48 \pm 0.03$ \\
15 & $0.70 \pm 0.01$ & $3.99 \pm 0.01$ & $8.35 \pm 0.02$ & $0.71 \pm 0.01$ & $0.73 \pm 0.01$ \\
16 & $0.45 \pm 0.02$ & $4.13 \pm 0.03$ & $7.99 \pm 0.01$ & $1.76 \pm 0.01$ & $0.99 \pm 0.01$ \\
17 & $0.56 \pm 0.01$ & $3.87 \pm 0.02$ & $11.06 \pm 0.01$ & $3.82 \pm 0.02$ & $2.45 \pm 0.05$ \\
18 & $0.56 \pm 0.02$ & $4.00 \pm 0.01$ & $11.82 \pm 0.02$ & $1.81 \pm 0.01$ & $1.91 \pm 0.01$ \\
19 & $0.88 \pm 0.01$ & $3.60 \pm 0.02$ & $10.42 \pm 0.02$ & $3.55 \pm 0.05$ & $3.82 \pm 0.02$ \\
20 & $0.77 \pm 0.03$ & $3.54 \pm 0.02$ & $13.25 \pm 0.05$ & $5.23 \pm 0.03$ & $1.30 \pm 0.02$ \\
21 & $0.56 \pm 0.01$ & $3.97 \pm 0.02$ & $7.91 \pm 0.01$ & $4.07 \pm 0.02$ & $1.99 \pm 0.01$ \\
22 & $0.38 \pm 0.00$ & $3.80 \pm 0.01$ & $13.69 \pm 0.04$ & $7.68 \pm 0.03$ & $1.53 \pm 0.03$ \\
23 & $0.91 \pm 0.01$ & $4.01 \pm 0.01$ & $9.23 \pm 0.03$ & $3.95 \pm 0.05$ & $0.92 \pm 0.02$ \\
\hline Mean & $0.58 \pm 0.018$ & $3.93 \pm 0.017$ & $10.11 \pm 0.020$ & $3.25 \pm 0.021$ & $2.15 \pm 0.020$ \\
\hline & & & & &
\end{tabular}

Table 2- Chemical properties of the hardaliye samples collected from different households

\begin{tabular}{cccccc}
\hline $\begin{array}{c}\text { Sample } \\
\text { no }\end{array}$ & $\begin{array}{c}\text { Total phenolic content } \\
\left(\mathrm{mg} \mathrm{L}^{-1}\right)\end{array}$ & $\begin{array}{c}\text { Monomeric anthocyanin } \\
\left(\mathrm{mg} \mathrm{L}^{-1}\right)\end{array}$ & $\begin{array}{c}\text { Volatile acids } \\
\left(\mathrm{g} \mathrm{L}^{-1}\right)\end{array}$ & $\begin{array}{c}\text { Ethanol } \\
(\%)\end{array}$ & $\begin{array}{c}\text { Total sugar } \\
(\%)\end{array}$ \\
\hline 1 & $1067.5 \pm 0.50$ & $54.4 \pm 0.03$ & $0.30 \pm 0.01$ & n.d & $18.0 \pm 0.10$ \\
2 & $1102.5 \pm 1.50$ & $44.6 \pm 0.10$ & $0.24 \pm 0.00$ & n.d & $17.6 \pm 0.10$ \\
3 & $2647.5 \pm 2.02$ & $118.4 \pm 0.11$ & $0.30 \pm 0.01$ & 1.6 & $15.9 \pm 0.02$ \\
4 & $2727.5 \pm 1.50$ & $109.1 \pm 0.10$ & $0.38 \pm 0.01$ & 1.3 & $15.8 \pm 0.07$ \\
5 & $1129.6 \pm 0.10$ & $29.0 \pm 0.01$ & $0.43 \pm 0.00$ & 0.9 & $17.7 \pm 0.01$ \\
6 & $861.8 \pm 0.08$ & $65.4 \pm 0.03$ & $0.26 \pm 0.01$ & n.d & $24.8 \pm 0.05$ \\
7 & $2318.9 \pm 1.53$ & $198.0 \pm 0.00$ & $0.24 \pm 0.00$ & n.d & $24.5 \pm 0.10$ \\
8 & $1693.9 \pm 1.00$ & $37.8 \pm 0.02$ & $0.50 \pm 0.01$ & n.d & $21.3 \pm 0.01$ \\
9 & $1111.8 \pm 0.10$ & $15.5 \pm 0.10$ & $0.42 \pm 0.01$ & n.d & $22.3 \pm 0.02$ \\
10 & $611.8 \pm 0.15$ & $55.0 \pm 0.53$ & $0.30 \pm 0.01$ & n.d & $24.1 \pm 0.02$ \\
11 & $922.50 \pm 1.50$ & $12.1 \pm 0.02$ & $0.54 \pm 0.01$ & 0.4 & $19.3 \pm 0.03$ \\
12 & $1336.8 \pm 0.40$ & $6.5 \pm 0.00$ & $0.36 \pm 0.00$ & n.d & $19.4 \pm 0.07$ \\
13 & $870.9 \pm 0.10$ & $39.5 \pm 0.20$ & $0.60 \pm 0.01$ & n.d & $24.3 \pm 0.10$ \\
14 & $583.1 \pm 0.02$ & $27.1 \pm 0.01$ & $0.36 \pm 0.01$ & n.d & $15.2 \pm 0.02$ \\
15 & $1636.8 \pm 0.03$ & $147.2 \pm 0.14$ & $0.50 \pm 0.01$ & 6.0 & $13.7 \pm 0.01$ \\
16 & $1129.6 \pm 0.04$ & $124.6 \pm 0.02$ & $0.24 \pm 0.01$ & n.d & $25.4 \pm 0.06$ \\
17 & $813.1 \pm 0.10$ & $4.5 \pm 0.03$ & $0.61 \pm 0.01$ & n.d & $20.8 \pm 0.05$ \\
18 & $576.60 \pm 0.18$ & $12.7 \pm 0.10$ & $0.48 \pm 0.00$ & n.d & $19.50 \pm 0.02$ \\
19 & $512.4 \pm 0.40$ & $11.6 \pm 0.05$ & $0.55 \pm 0.01$ & n.d & $23.1 \pm 0.03$ \\
20 & $908.8 \pm 0.10$ & $27.9 \pm 0.10$ & $0.72 \pm 0.01$ & 2.1 & $7.2 \pm 0.01$ \\
21 & $1311.8 \pm 0.09$ & $71.9 \pm 0.04$ & $0.38 \pm 0.00$ & n.d & $18.6 \pm 0.04$ \\
22 & $368.8 \pm 0.10$ & $23.4 \pm 0.01$ & $0.30 \pm 0.01$ & n.d & $24.4 \pm 0.09$ \\
23 & $890.4 \pm 0.30$ & $103.2 \pm 0.08$ & $0.54 \pm 0.01$ & 1.0 & $12.9 \pm 0.02$ \\
\hline Mean & $1179.7 \pm 0.515$ & $55.5 \pm 0.080$ & $0.41 \pm 0.007$ & & $19.4 \pm 0.046$ \\
\hline
\end{tabular}

${ }^{+}$, Monomeric anthocyanidin (malvidin-3-glocoside); n.d, not detected. Note: Ethanol values are not included in the statistical analysis 
Table 3- Microbiological properties of the hardaliye samples collected from different households (cfu mL $\left.{ }^{-1}\right)$

\begin{tabular}{cccc}
$\begin{array}{c}\text { Sample } \\
\text { no }\end{array}$ & T.M.A.B. & $\begin{array}{c}\text { LAB } \\
\text { (Lactobacilli) }\end{array}$ & Yeast-Molds \\
\hline 1 & $4.00 \times 10^{3} \pm 1 \times 10^{2}$ & $1.00 \times 10^{2} \pm 0.00$ & $<10 \pm 0.00$ \\
2 & $3.00 \times 10^{1} \pm 0.6 \times 10^{1}$ & $<10 \pm 0.00$ & $2.30 \times 10^{4} \pm 1.5 \times 10^{3}$ \\
3 & $6.00 \times 10^{3} \pm 1 \times 10^{3}$ & $4.00 \times 10^{3} \pm 2 \times 10^{2}$ & $3.30 \times 10^{3} \pm 5.7 \times 10^{1}$ \\
4 & $1.19 \times 10^{6} \pm 5 \times 10^{3}$ & $1.00 \times 10^{2} \pm 0.00$ & $<10 \pm 0.00$ \\
5 & $1.15 \times 10^{5} \pm 1 \times 10^{3}$ & $1.80 \times 10^{4} \pm 4 \times 10^{2}$ & $1.00 \times 10^{4} \pm 1 \times 10^{2}$ \\
6 & $3.00 \times 10^{5} \pm 1 \times 10^{4}$ & $4.10 \times 10^{4} \pm 1.5 \times 10^{3}$ & $1.00 \times 10^{1} \pm 0.1 \times 10^{1}$ \\
7 & $3.20 \times 10^{6} \pm 2 \times 10^{5}$ & $<10 \pm 0.00$ & $2.00 \times 10^{1} \pm 0.1 \times 10^{1}$ \\
8 & $3.00 \times 10^{3} \pm 0.00$ & $9.00 \times 10^{2} \pm 1 \times 10^{2}$ & $1.20 \times 10^{3} \pm 1 \times 10^{2}$ \\
9 & $2.00 \times 10^{3} \pm 2 \times 10^{2}$ & $1.60 \times 10^{3} \pm 0.00$ & $1.00 \times 10^{4} \pm 1 \times 10^{2}$ \\
10 & $4.00 \times 10^{3} \pm 1 \times 10^{2}$ & $3.00 \times 10^{2} \pm 1 \times 10^{1}$ & $2.00 \times 10^{2} \pm 1 \times 10^{1}$ \\
11 & $2.00 \times 10^{3} \pm 1 \times 10^{2}$ & $<10 \pm 0.00$ & $2.00 \times 10^{2} \pm 1 \times 10^{1}$ \\
12 & $2.00 \times 10^{5} \pm 3 \times 10^{3}$ & $1.00 \times 10^{5} \pm 1 \times 10^{1}$ & $7.00 \times 10^{2} \pm 1 \times 10^{1}$ \\
13 & $2.00 \times 10^{5} \pm 2 \times 10^{3}$ & $1.70 \times 10^{5} \pm 3 \times 10^{3}$ & $<10 \pm 0.00$ \\
14 & $5.00 \times 10^{5} \pm 1 \times 10^{4}$ & $3.00 \times 10^{5} \pm 5 \times 10^{3}$ & $4.00 \times 10^{1} \pm 0.1 \times 10^{1}$ \\
15 & $1.00 \times 10^{5} \pm 0.00$ & $3.20 \times 10^{3} \pm 1 \times 10^{2}$ & $<10 \pm 0.00$ \\
16 & $1.00 \times 10^{5} \pm 1 \times 10^{3}$ & $1.00 \times 10^{3} \pm 1 \times 10^{2}$ & $1.00 \times 10^{1} \pm 0.1 \times 10^{1}$ \\
17 & $3.00 \times 10^{4} \pm 3 \times 10^{3}$ & $1.00 \times 10^{4} \pm 1 \times 10^{2}$ & $6.60 \times 10^{2} \pm 1 \times 10^{1}$ \\
18 & $2.00 \times 10^{4} \pm 4 \times 10^{2}$ & $1.20 \times 10^{4} \pm 1 \times 10^{3}$ & $7.00 \times 10^{2} \pm 6.1 \times 10^{1}$ \\
19 & $1.00 \times 10^{5} \pm 2 \times 10^{3}$ & $<10 \pm 0.00$ & $2.50 \times 10^{3} \pm 1 \times 10^{2}$ \\
20 & $3.00 \times 10^{4} \pm 1 \times 10^{3}$ & $<10 \pm 0.00$ & $3.80 \times 10^{3} \pm 1 \times 10^{2}$ \\
21 & $3.00 \times 10^{4} \pm 1 \times 10^{3}$ & $<10 \pm 0.00$ & $<10 \pm 0.00$ \\
22 & $4.00 \times 10^{4} \pm 3 \times 10^{3}$ & $<10 \pm 0.00$ & $4.00 \times 10^{2} \pm 1 \times 10^{1}$ \\
23 & $2.60 \times 10^{4} \pm 1 \times 10^{3}$ & $1.50 \times 10^{2} \pm 1 \times 10^{1}$ & $1.90 \times 10^{4} \pm 5 \times 10^{2}$ \\
\hline Mean & $2.70 \times 10^{5} \pm 1.07 \times 10^{4}$ & $2.90 \times 10^{4} \pm 5.46 \times 10^{2}$ & $3.30 \times 10^{3} \pm 1.17 \times 10^{2}$ \\
\hline
\end{tabular}

cfu, colony forming units

Table 4- Physicochemical properties of the hardaliye samples produced in laboratory

\begin{tabular}{|c|c|c|c|c|c|c|}
\hline \multirow{2}{*}{ Trials } & \multirow{2}{*}{ Grape variety } & \multirow{2}{*}{ Acidity (\%) } & \multirow{2}{*}{$p H$} & \multicolumn{3}{|c|}{ Color } \\
\hline & & & & $L$ & $a$ & $b$ \\
\hline 1 & Dingil Kara & $0.73 \pm 0.02^{\mathrm{b}}$ & $3.66 \pm 0.02^{\mathrm{d}}$ & $9.24 \pm 0.02^{\mathrm{c}}$ & $2.89 \pm 0.04^{\mathrm{a}}$ & $1.27 \pm 0.01^{\mathrm{c}}$ \\
\hline 2 & Kara Üzüm & $1.05 \pm 0.04^{\mathrm{e}}$ & $3.47 \pm 0.32^{\mathrm{b}}$ & $9.71 \pm 0.01^{\mathrm{d}}$ & $3.69 \pm 0.02^{\mathrm{c}}$ & $1.04 \pm 0.03^{b}$ \\
\hline 3 & Cabernet Sauvignon* & $0.56 \pm 0.01^{\mathrm{a}}$ & $3.73 \pm 0.03^{\mathrm{e}}$ & $8.45 \pm 0.05^{\mathrm{a}}$ & $4.20 \pm 0.04^{\mathrm{d}}$ & $1.89 \pm 0.01^{\mathrm{d}}$ \\
\hline 4 & Yerli İzabella** & $0.77 \pm 0.01^{\mathrm{c}}$ & $3.33 \pm 0.03^{\mathrm{a}}$ & $9.86 \pm 0.02^{\mathrm{e}}$ & $3.13 \pm 0.03^{\mathrm{b}}$ & $1.28 \pm 0.01^{\mathrm{c}}$ \\
\hline 5 & Siyah Üzüm & $0.98 \pm 0.02^{\mathrm{d}}$ & $3.50 \pm 0.06^{\mathrm{c}}$ & $8.75 \pm 0.05^{\mathrm{b}}$ & $5.00 \pm 0.03^{\mathrm{e}}$ & $0.86 \pm 0.02^{\mathrm{a}}$ \\
\hline
\end{tabular}

Note: The analyzes were carried out at the end of the fermentation. The different letters in the form of upper indices indicate that the averages are different $(\mathrm{P}<0.01)$ according to the Duncan Multiple Comparison Test. *, Cabernet Sauvignon; **, Yerli İzabella

Table 5- Chemical properties of the hardaliye samples produced in laboratory

\begin{tabular}{clccccc}
\hline Trials & Grape variety & $\begin{array}{c}\text { Total phenolic } \\
\text { content } \\
\left(m g L^{-1}\right)\end{array}$ & $\begin{array}{c}\text { Monomeric } \\
\text { anthocyanin } \\
\left.L^{-1}\right)\end{array}$ & $\begin{array}{c}\text { Volatile acids } \\
\left(\mathrm{g} \mathrm{L}^{-1}\right)\end{array}$ & $\begin{array}{c}\text { Ethanol } \\
(\%)\end{array}$ & $\begin{array}{c}\text { Total sugar } \\
(\%)\end{array}$ \\
\hline 1 & Dingil Siyah & $2286.8 \pm 2.15^{\mathrm{e}}$ & $210.8 \pm 1.33^{\mathrm{e}}$ & $0.43 \pm 0.01^{\mathrm{c}}$ & $\mathrm{n}^{\mathrm{c}} \mathrm{d}^{*}$ & $16.5 \pm 0.4^{\mathrm{b}}$ \\
2 & Kara Üzüm & $1858.2 \pm 0.20^{\mathrm{d}}$ & $116.3 \pm 1.3^{\mathrm{a}}$ & $0.55 \pm 0.02^{\mathrm{d}}$ & n.d & $15.2 \pm 0.3^{\mathrm{a}}$ \\
3 & Cabernet Sauvignon** & $1165.4 \pm 1.40^{\mathrm{c}}$ & $200.6 \pm 0.3^{\mathrm{c}}$ & $0.42 \pm 0.02^{\mathrm{bc}}$ & n.d & $19.7 \pm 0.4^{\mathrm{d}}$ \\
4 & Yerli İzabella*** & $1093.9 \pm 0.50^{\mathrm{b}}$ & $201.3 \pm 1.30^{\mathrm{d}}$ & $0.39 \pm 0.01^{\mathrm{a}}$ & n.d & $21.4 \pm 0.3^{\mathrm{e}}$ \\
5 & Siyah Üzüm & $961.8 \pm 0.40^{\mathrm{a}}$ & $161.1 \pm 1.00^{\mathrm{b}}$ & $0.40 \pm 0.02^{\mathrm{ab}}$ & n.d & $17.9 \pm 0.2^{\mathrm{c}}$ \\
\hline
\end{tabular}

${ }^{+}$, Monomeric anthocyanin (malvidin-3-glucoside); ${ }^{*}$, n.d, not detected; **, Cabernet Sauvignon; ***, Yerli İzabella. Note: Ethanol values are not included in the statistical analysis 
Table 6- Microbiological properties of the hardaliye samples produced in laboratory (cfu $\mathrm{mL}^{-1}$ )

\begin{tabular}{clccc}
\hline Trials & Grape variety & TMAB $^{+}$ & LAB $^{-}$(Lactobacilli) & Yeast-Molds \\
\hline 1 & Dingil Kara & $7.00 \times 10^{6} \pm 1 \times 10^{6} \mathrm{~b}$ & $1.68 \times 10^{6} \pm 1 \times 10^{4} \mathrm{e}$ & $<10 \pm 0.00^{\mathrm{a}}$ \\
2 & Kara üzüm & $1.60 \times 10^{7} \pm 2 \times 10^{6} \mathrm{c}$ & $2.20 \times 10^{5} \pm 2 \times 10^{4 \mathrm{~d}}$ & $1.00 \times 10^{2} \pm 0.1 \times 10^{1 \mathrm{c}}$ \\
3 & Cabernet Sauvignon* & $2.00 \times 10^{5} \pm 1 \times 10^{4} \mathrm{a}$ & $1.00 \times 10^{2} \pm 0.1 \times 10^{1 \mathrm{a}}$ & $3.50 \times 10^{1} \pm 0.00^{\mathrm{b}}$ \\
4 & Yerli İzabella & $8.16 \times 10^{5} \pm 6 \times 10^{3 \mathrm{a}}$ & $2.76 \times 10^{4} \pm 3 \times 10^{2 \mathrm{c}}$ & $<10 \pm 0.00^{\mathrm{a}}$ \\
5 & Siyah üzüm & $1.00 \times 10^{5} \pm 1 \times 10^{3 \mathrm{a}}$ & $3.00 \times 10^{3} \pm 3 \times 10^{2 \mathrm{~b}}$ & $<10 \pm 0.00^{\mathrm{a}}$ \\
\hline
\end{tabular}

${ }^{+}$TMAB, total mesophilic aerobic bacteria; -LAB, Lactic acid bacteria; cfu, colony forming units; *, Cabernet Sauvignon

the amount of these components to decrease. For this reasons, total phenolics of anthocyanins of hardaliye samples collected from producers may be lower than others. Coşkun et al (2012) determined that the amount of monomeric anthocyanins (malvidin-3-glucoside) and total phenolics of hardaliye samples decreased from 114.1 to $54.4 \mathrm{mg} \mathrm{L}^{-1}$ and from 1392.5 to 1067.5 $\mathrm{mg} \mathrm{L}^{-1}$ respectively, during 1 year of storage. Zarfilla et al (2003) produced wine from red grapes grown organically and traditionally in Spain and stored in glass bottles in a dark environment for 7 months at $20{ }^{\circ} \mathrm{C}$. They observed a decrease of approximately $65 \%$ over 7 months in the total amount of total phenolics. In the same study, the monomeric anthocyanins (malvidin-3-glucoside) decreased from $248.34 \mathrm{mg} \mathrm{L}^{-1}$ to $32.29 \mathrm{mg} \mathrm{L}^{-1}$ in the conventional red grape wine, from $228.5 \mathrm{mg}$ $\mathrm{L}^{-1}$ to $22.45 \mathrm{mg} \mathrm{L}^{-1}$ in the organic red grape wine. Hardaliye was produced from papazkarası blueblack grapes by conventional method by Aşkın \& Atik (2016). After having been exposed to lactic acid fermentation, it was bottled and then stored at $+4{ }^{\circ} \mathrm{C}$ and $20^{\circ} \mathrm{C}$ for 60 days. The analyses were carried out in prepared beverage within the 15 , 30,45 and 60 days of storage. The results of color parameters obtained show the highest proportion of red color in the samples at the beginning $(\mathrm{dA} \%=$ 94.87). As expected, the brown color increased with storage time and the highest value was determined for 60 days depending on the storage temperature. Storage under $4{ }^{\circ} \mathrm{C}$ and $20{ }^{\circ} \mathrm{C}$ resulted in 60 and $78 \%$ losses in anthocyanin content, respectively.

In the present study, ethyl alcohol was found in some hardaliye samples collected from different households. The reason might be that preservatives such as benzoic acid and mustard seeds were not used at the sufficient amount. Moreover, improper storage conditions and prolonged storage time increases formation of alcohol.

In the study conducted by Coşkun et al (2009), hardaliye sample was produced using black grape from Malatya region's grape varieties by applying traditional. Total mesophilic aerobic bacteria count in the hardaliye sample was lower $\left(4.7 \times 10^{4}\right)$ than those in the samples produced in laboratory in this study. This may be caused by harvesting and fermentation conditions.

The results of this study were also in accordance with the study conducted by Arici \& Coskun (2001) in which red colour (measured by Hunter Lab) of hardaliye samples ranged from 1.33 to 9.66 and total mesophilic aerobic bacteria, lactic acid bacteria and yeasts-molds counts ranged from $3.5 \times 10^{2}$ to $8 \times 10^{5}$ cfu $\mathrm{mL}^{-1}, 1.0 \times 10^{2}$ to $4.0 \times 10^{4} \mathrm{cfu} \mathrm{mL}^{-1}$ and $1.0 \times 10^{2}$ to $8.1 \times 10^{4} \mathrm{cfu} \mathrm{mL}^{-1}$, respectively. Furthermore, coliforms and E. coli were not found in none of the samples.

Güven \& Aksoy (2009) produced hardaliye using verigo variety of grapes. $2.5 \mathrm{~g}$ mustard seeds were used. Hardaliye was fermented for 21 day. On the $7^{\text {th }}$ and $21^{\text {st }}$ days of fermentation, $\mathrm{pH}$ and total acidity were detected to range from 4.17 to 3.94 and 3.39 to $10.40 \%$, respectively. They found higher $\mathrm{pH}$ value than the present study, however, total acidity was comparable. They did not detect ethyl alcohol which was similar to the hardaliye samples produced by applying traditional method in the present study. 
Kilic \& Copur (1988) used Muskule, Razaki and Erenkoy Beyazı as grape varieties in their research. The total sugar content of hardaliye produced from these varieties was determined as 12.92, 14.94 and

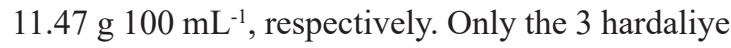
samples from the present study were in accordance with Kılıç \& Çopur (1988)'s observation. In this study, the total sugar contents of hardaliye samples were detected to range from $7.2 \%$ to $25.4 \%$, which may be attributed to different grape varieties and maturities. The total amount of sugar in the grapes increased towards end of the maturity period (Çelik 1998). The grape harvest for hardaliye production usually starts on October or November. The hardaliye samples with high sugar content may be produced from late harvested grapes. The hardaliye samples with low sugar content may be produced from early harvested grapes. The formation of alcohol due to uncontrolled fermentation or improper storage conditions may have caused to decrease of sugar content.

\section{Conclusions}

There are very few scientific studies about traditional hardaliye beverage although it has been produced traditionally for years. It brings the opportunity of consuming grapes in winter or spring seasons; thus, preventing the excess grapes from being wasted. Just like any other popular traditional food products, industrial-scale production of hardaliye should be realized and this traditional beverage should enjoy the large number of consumers' preference. In addition, more research is needed in order to its adaptation to industry.

\section{Acknowledgements}

The authors gratefully acknowledged the TUBITAK (Project number: 1100018) for providing funding for this work.

\section{References}

Anonymous (2001a). Aerobic Plate Count, FDA Bacteriological Analytical Manual, Chap. 3
Anonymous (2001b). Staphylococcus aureus, FDA Bacteriological Analytical Manual, Chap. 12

Anonymous (2001c). Yeasts, Molds and Mycotoxins, FDA Bacteriological Analytical Manual, Chap. 18

Anonymous (2002). Enumeration of E. coli and the Coliform Bacteria, FDA Bacteriological Analytical Manual, Chap. 4

Arici M \& Coskun F (2001). Hardaliye: fermented grape juice as a traditional Turkish beverage. Food Microbiology 18: 417-421

Aşkın B \& Atik A (2016). Color and antioxidant properties of fermented grape beverage under different storage conditions. Turkish Journal of Agriculture and Forestry 40: 803-812

Baumgart J (1993). Mikrobiologische Untersuchung von Lebensmitteln, Behr's Verlag, Hamburg

Cemeroğlu B (2007). Gıda Analizleri. G1da Teknolojisi Derneği Yayınları No: 34, Ankara, Turkey

Coşkun F, Arici M, Gülcü M \& Çelikyurt G (2009). Some physicochemical, microbiological and sensory properties of Hardaliye produced using different methods In: II. Traditional Foods Symposium, 27-29 May, Van, Turkey, pp. 665-669

Coşkun F, Arici M, Çelikyurt G \& Gülcü M (2012). Changes occuring at the end of storage in some properties of hardaliye produced by using different methods. Journal of Tekirdag Agricultural Faculty 9(3): 62-67

Çelik S (1998). Asmanın Büyüme ve Gelişme Fizyolojisi, Bağcılık (Ampeleloji) Cilt 1. 426 s, Tekirdag, Turkey

Fidan I (1975). Şarap Analiz Yöntemleri. Tekel Enstitüleri Yayınları, Seri A No: 11, Istanbul, Turkey

Güven S \& Aksoy M (2009). Some Modifications in Hardaliye Production. In: II. Traditional Foods Symposium, 27-29 May, Van, Turkey, pp. 675-678

Jacobson J L (2006). Introduction to Wine Laboratory Practices and Procedures. Springer Science and Business Media New York, pp. 375

Kanemaru K \& Miyamoto T (1990). Inhibitory effects on the growth of several bacteria by brown mustard and allyl isothiocyanalte. Journal of Japanese Society Food Science and Technology 37: 823- 829

Kilıç O \& Çopur Ö U (1988). The quality criteria of hardaliye produced from some grape varieties In: Turkey III. Viticulture Symposium, 31 May-3 June, Bursa, Turkey, pp. 12 
Lemay M J, Choquette J, Delaquis P J, Gariépy C, Rodrigue N \& Saucier L (2002). Antimicrobial effect of natural preservatives in a cooked and acidified chicken meat model. International Journal of Food Microbiology 78: 217-226

Middleton E J (1998). Effect of plant flavonoids on immune and inflammatory cell function. Advances in Experimental Medicine and Biology 439: 175-182

Nielsen P V \& Rios R (2000). Inhibition of fungal growth on bread by volatile components from spices and herbs, and the possible application in active packaging, with special emphasis on mustard essential oil. International Journal of Food Microbiology 60: 219-229
Shahidi F \& Naczk M (1995). Food phenolics, sources, chemistry, effects, applications. Technomic publishing company, Inc. USA

Singleton V L \& Rossi J A (1965). Colorimetry of total phenolics with phosphomolybdic-phosphotungstic acid reagents. American Journal of Enology and Viticulture 16: 144-158

SPSS 2009. PASW Statistics for Windows, Version 18.0. Chicago: SPSS Inc

Zarfilla P, Morillas J, Mulero J, Cayuela J M, MartinezCacha A, Pardo F \& Lopez Nicolas J M (2003). Changes during Storage in Conventional and Ecological Wine: Phenolic Content and Antioxidant Activity. Journal of Agricultural Food Chemistry 51(16): 4694-4700 